\title{
New and emerging technology in the diagnosis and treatment of pancreatic cysts
}

\author{
Lindsey C. Shipley ${ }^{1}$, Ali M. Ahmed ${ }^{2}$ \\ ${ }^{1}$ Department of Internal Medicine, University of Alabama, Birmingham, AL, USA; ${ }^{2}$ Division of Gastroenterology and Hepatology, University of \\ Alabama, Birmingham, AL, USA \\ Contributions: (I) Conception and design: Both authors; (II) Administrative support: AM Ahmed; (III) Provision of study materials or patients: Both \\ authors; (IV) Collection and assembly of data: Both authors; (V) Data analysis and interpretation: Both authors; (VI) Manuscript writing: Both \\ authors; (VII) Final approval of manuscript: Both authors. \\ Correspondence to: Ali M. Ahmed, MD. Division of Gastroenterology and Hepatology, University of Alabama at Birmingham, $17202^{\text {nd }}$ Avenue South, \\ BDB 380, Birmingham, AL 35294, USA. Email: amahmed@uabmc.edu.
}

\begin{abstract}
Pancreatic cysts have always presented as a diagnostic dilemma due to the difficulties in identifying patients with current imaging modalities that could most benefit from surgical intervention. Intraductal papillary mucinous neoplasms (IPMNs) and mucinous cystadenomas (MCNs) carry the highest malignant potential of all pancreatic cysts and pancreatic adenocarcinoma carries a high mortality as the fourth leading cause of cancer-related deaths. However, surgery to remove benign cysts also carries a high morbidity and occasional mortality. Opportunities to identify and reduce pre-cancer lesions must be aggressively pursued. Multidetector helical CT (MDHCT) or an up-to-date MRI is the first diagnostic tool to evaluate a suspected pancreatic lesion. Currently, review by a multidisciplinary group who specialize in pancreatic cysts and pancreatic cancer is advised to review factors such as a patient's comorbidities, the type of surgery needed to remove the cyst and the estimated morbidity and mortality associated with the procedure. Some recent data are emerging to assist with identifying those at highest risk such as cyst fluid analysis, laser endomicroscopy, and artificial intelligence (AI). This article reviews the current status, benefits, challenges and future prospects on diagnosis and treatment of pancreatic cysts. Further prospective randomized control trials are needed to determine the optimal management and treatment for patients with pancreatic cysts.
\end{abstract}

Keywords: Pancreatic cyst; intraductal papillary mucinous neoplasm (IPMN); pancreatic adenocarcinoma; endoscopic ultrasound; artificial intelligence (AI)

Received: 14 December 2019; Accepted: 05 June 2020; Published: 25 April 2022.

doi: 10.21037/tgh-2020-09

View this article at: http://dx.doi.org/10.21037/tgh-2020-09

\section{Introduction}

The incidence of pancreatic cysts increases with age and is approximately $25 \%$ in those greater than 70 years of age (1). The prevalence of pancreatic cysts was $2.4 \%$ in a large study of 2,803 patients who underwent MRI with only a minority of cysts (nine) being greater than $2 \mathrm{~cm}$ (2). The types of pancreatic cysts include serous cystadenomas, mucinous cystadenomas (MCNs), solid papillary neoplasms, cystic degeneration of adenocarcinomas, neuroendocrine tumors and side branch or main branch intraductal papillary mucinous neoplasms (IPMNs) and each infer different malignant potential (1). Pancreatic ductal adenocarcinoma (PDAC) is the fourth leading cause of cancer-related mortality, is the most deadly of all gastrointestinal malignancies. Furthermore, PDAC carries a poor prognosis with less than a 5\%, 5-year survival rate (3). Pancreatic cysts are being increasingly diagnosed as abdominal imaging both improves and is being utilized more commonly. Management decision for asymptomatic pancreatic cysts must consider risk for malignancy as the risk of pancreatic surgery has a mortality of $2.1 \%$ and morbidity of $30 \%$ (4). Pancreatic cysts have always presented as a diagnostic 
dilemma due to the difficulties to identify patients with current imaging modalities that could most benefit from surgical intervention (3). This review will discuss both current diagnostic and therapeutic approaches and well as emerging diagnostics and therapies for pancreatic cysts.

\section{Current diagnostic modalities}

Cystic lesions most at risk for malignant conversion are IPMNs, MCNs, solid pseudopapillary tumors and pancreatic neuroendocrine tumors. Current diagnostics rely on advanced cross-sectional imaging such as computed tomography (CT), magnetic resonance imaging (MRI) and cysts fluid analysis (4). A well trained radiologist is needed to identify high-risk stigmata on imaging. Worrisome features include a cyst $>3 \mathrm{~cm}$, enhancing mural nodule $<5 \mathrm{~mm}$, thickened/enhanced walls, non-enhanced mural nodules and a main pancreatic duct $5-9 \mathrm{~mm}$ or a rapid rate of cyst growth $>5 \mathrm{~mm} / 2$ years. High-risk stigmata include extrahepatic biliary obstruction by a pancreatic head cyst, an enhanced solid component and a main pancreatic duct greater than $10 \mathrm{~mm}$ without other cause of obstruction $(5,6)$.

\section{$C T$}

Multidetector helical CT (MDHCT) is a dedicated pancreatic protocol scanner that obtains submillimeter slices $(0.5-1 \mathrm{~mm})$ to allow for optimal 3D visualization. Pancreatic-phase images begin approximately 50 seconds after intravenous contrast injection for peri-pancreatic vasculature and enhancement with a second phase at approximately 80 seconds to evaluate the liver (5). MDHCT is the preferred method for initial evaluation in patients for whom a pancreatic lesion is suspected (7). This modality carries a reported sensitivity of $97-100 \%$ and nonresectability prediction near $100 \%$ for pancreatic cancer lesions (3). However, in a retrospective study of 36 patients, the overall sensitivity, specificity and accuracy of MDHCT to discriminate benign versus malignant was $36.3 \%, 100 \%$ and $78.8 \%$, respectively (8).

\section{MRI}

MRI has been shown to be equally sensitive and specific in staging pancreatic cancer and can be used interchangeably however, due to cost and availability is not commonly utilized (7). The reported sensitivity of MRI to detect pancreatic cancer ranges from $83 \%$ to $87 \%$ with a specificity of $81 \%$ to $100 \%$ (3). However, in a study of 136 resected patients with incidental pancreatic cysts on cross sectional imaging (CT, MRI or both) diagnosis was only correct in $63 \%$ of cases and $20 \%$ of branch duct-IPMN had a main duct component $(3,9)$. MRI uses fat-suppressed T2 weighted images and gadolinium enhanced T1 weighted sequences in the arterial, early portal and later portal phases to evaluate pancreatic cystic lesions. In some cases, magnetic resonance cholangiopancreatography (MRCP) can help delineate cyst communication with the main pancreatic duct (5). Based on an analysis from 2005-2015 of 500 patients, the new version of MRI hardware could detect pancreatic cysts in $56.3 \%$ of patients as compared to the $30.3 \%$ detection rate of older hardware (10).

\section{Endoscopic ultrasound}

MDHCT or an up-to-date MRI is the first diagnostic tool to evaluate a suspected pancreatic lesion, however, endoscopic ultrasound can be useful as a second diagnostic tool in several cases (3). EUS can detect small lesions that are difficult to visualize on cross-sectional imaging and can assess morphology, cyst fluid analysis and cytopathology with an EUS-guided fine needle aspiration (11). EUS should be considered in negative results on CT/MRI and persistent strong clinical suspicion, doubtful results on CT/MRI or need for cyto-histological confirmation. EUS has shown to be useful to assess a more accurate lesion size, especially those lesions less than $2 \mathrm{~cm}$, and lymph node involvement (3). EUS coupled with cyst fine needle aspiration (FNA) has been used with increasing frequency to determine if a cyst is mucinous, connects to the main duct or assess mutations in the cyst fluid that may predict future malignant potential (5). The presence of non-gut mucin and carcinoembryonic antigen greater than $192 \mathrm{ng} / \mathrm{mL}$ can identify a mucinous cyst and only requires two milliliters of fluid to perform the analysis (5).

\section{Current recommendations}

\section{American Gastrointestinal Association (AGA) guidelines (1)}

AGA guidelines were created due to the lack of evidence in current clinical practice when asymptomatic mucinous cysts are discovered on imaging. Their recommendations advocate for less frequent surveillance and a higher threshold to proceed with EUS and surgery. Furthermore, these guidelines do not discuss symptomatic cysts or some neoplastic lesions such as solid papillary neoplasms, cystic 
degeneration of adenocarcinomas, neuroendocrine tumors and main duct IPMNs without side branch involvement.

Per recommendations, pancreatic cysts $<3 \mathrm{~cm}$ without a solid component or dilated PD should undergo MRI for surveillance at 1 year and then every 2 years until reaching 5 years if there is no growth of the cyst. However, those with 2 or more high-risk features (size $>3 \mathrm{~cm}$, a dilated main PD, or a solid component) should be examined with EUSFNA. If there is nothing concerning on EUS-FNA, patient should switch back to MRI surveillance at 1 year then 2 years to ensure no changes. If there are significant changes in the cyst (high-risk features as above), EUS-FNA should be performed. However, if there are no significant changes in the cyst after 5 years of surveillance or the patient is not a surgical candidate, surveillance should stop. Whether cessation of surveillance after 5 years is safe is not based on clinical evidence and remains are area of debate (6). Surgery should be offered to those with both a solid component and a dilated PD and/or concerning features discovered on EUS-FNA. Post-surgical procedure surveillance should be performed with MRI of the remaining pancreas every 2 years.

\section{American College of Radiology (ACR) guidelines (5)}

ACR guidelines were created by the Pancreas Subcommittee made up of an abdominal radiologist, a gastroenterologist and pancreatic surgeon and are recommendations for managing incidental pancreatic cysts discovered on advanced imaging such as CT or MRI. As compared to the AGA guidelines, these recommendations advocate for more frequent surveillance of pancreatic cysts however, they address more types of pancreatic cysts than the AGA guidelines.

Per recommendations, incidental pancreatic cysts found to be less than $1.5 \mathrm{~cm}$ are further subdivided by patient age (above/below) 65 years at presentation. If less than 65, the recommendation is to reimage every 1 year for 5 years. If the lesions remain stable over 5 years, surveillance can be increased to every 2 years for 2 times. If stable, surveillance may stop. For any interval growth of lesions less than $1.5 \mathrm{~cm}$ the recommendation is to reimage every 1 year or EUS/ FNA. However, if the lesion becomes greater than $1.5 \mathrm{~cm}$, then EUS/FNA should be performed. For ages 65-79, the ACR recommends reimaging every 2 years and stopping at 10 years if stable. For interval growth but the lesion size remains less than $1.5 \mathrm{~cm}$, the recommendation is to reimage annually or perform EUS/FNA. Again, if the lesion becomes greater than $1.5 \mathrm{~cm}, \mathrm{EUS} / \mathrm{FNA}$ is recommended.

Incidental pancreatic cysts of intermediate size (1.5-
$2.5 \mathrm{~cm}$ ) with MPD communication established by imaging can be evaluated by EUS/FNA. However, there is an option for surveillance for lesions $(1.5-1.9 \mathrm{~cm})$ with repeat imaging annually for 5 years then every 2 years for two times until stable for 9 years. If any interval growth but the cyst is still less than $2.5 \mathrm{~cm}$ increase the imaging surveillance frequency to every 6 months or perform an EUS/FNA, if the cyst is $>2.5 \mathrm{~cm}$ perform an EUS/FNA. If the initial lesion is 2.0 $2.5 \mathrm{~cm}$, reimage every 6 months for 4 times then annually twice followed by every 2 years for three times and then stop if the lesion is stable over 10 years. For any interval growth, perform EUS/FNA.

If the lesion is $1.5-2.5 \mathrm{~cm}$ and MPD communication cannot be determined or is not present, the ACR recommends reimaging every 6 months for four times or EUS/FNA. If pathology is concerning for mucinous cyst or indeterminate cyst, reimagine every 6 months for 2 years then annually for the next 2 years and then every 2 years for three times. For any interval growth, consider surgical consultation.

If the incidental pancreatic cyst is $>2.5 \mathrm{~cm}$ one can proceed with EUS/FNA or classify as low risk or high risk based upon imaging. High risk lesions should be evaluated by EUS/FNA and surgical consultation. For low risk cysts, image every 6-month for 2 years followed by annually for two years then every 2 years for three times. Surveillance can be stopped if the cyst is stable over 10 years but for any interval growth, proceed with EUS/FNA and surgical consultation.

\section{Revision of Consensus Fukuoka guidelines (6)}

These guidelines are more aggressive to consider surgery in the surgically fit patient and do not comment on cessation of surveillance. The rationale for this is that the malignant progression of IPMN is not reduced over time. The guidelines recommend surgery for any worrisome features (obstructive jaundice in a patient with pancreatic head cystic lesion, enhancing mural nodule larger than $5 \mathrm{~mm}$ and main pancreatic duct greater than $10 \mathrm{~mm}$ ) in the clinically appropriate situation. Surveillance with EUS is recommended for intermediate lesions and further follow is then based upon cyst size.

Our institution employs the strategies from the above guidelines in a customized version involving multidisciplinary evaluation at a weekly pancreatobiliary disease conference. At this conference patient symptoms, health status, fitness/willingness for surveillance/surgery 


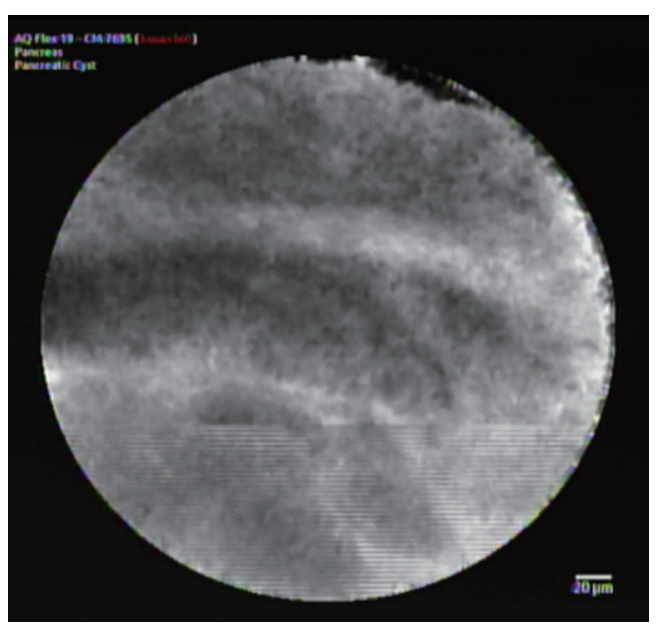

Figure 1 Confocal laser endomicroscopy demonstrating multiple layers of epithelial bands. (Source: Ali M. Ahmed MD, 2019).

along with endoscopy/radiographic findings are reviewed to determine optimal patient care. Our approach is summarized in Figure S1.

\section{Emerging diagnostics}

\section{Mutational analysis of cyst fluid}

Mutation analysis includes K-ras, GNAS, mRNA 21 and glucose and cytology can reveal dysplastic cells (5,12-14). Pancreatic cyst fluid DNA analysis (PANDA) trial was a prospective multi-center study that demonstrated cyst fluid $\mathrm{k}$-ras mutation was helpful in the diagnosis of mucinous cysts (OR 20.9, specificity 96\%). It also demonstrated high amounts of pancreatic cyst fluid DNA and high-amplitude mutations are indicators of malignancy (15). However, this was a study in which all patients were subject to surgical intervention. A randomized controlled trial of patients with long-term follow-up who are not subject to surgical intervention is needed to determine value of these tests (16). A recent retrospective study of 130 patients identified molecular markers and clinical features that classified cysts type with $90-100 \%$ sensitivity and $92-98 \%$ specificity. Furthermore, their data identified patients who did not require surgery and reduced the number of unnecessary operations by $91 \%$ (17).

\section{Needle-based confocal laser endomicroscopy (nCLE)}

Confocal laser endomicroscopy is an endoscopic modality used to obtain high-resolution images of the mucosal layer of the GI tract. nCLE uses tissue illumination via a lowpower laser based on tissue fluorescence allowing for high quality images comparable to traditional histology. In this procedure, the EUS needle is used to access the cyst by FNA with a 19-g needle and then the confocal probe is placed through the working channel of the echoendoscope and under EUS guidance placed on the cyst wall where certain patterns suggest differing cyst times (18). Figure 1 depicts the nCLE images of multiple epithelial bands observed in a mucinous cystic neoplasm. Current studies have focused on pancreatic tissue disease, including pancreatic cystic neoplasms. Two studies looking at the use of nCLE for the diagnosis of pancreatic cystic neoplasms produced a sensitivity of $68 \%$ and specificity of $90 \%$ (19-21). EUS-guided nCLE is minimally invasive with excellent potential for diagnosis however, further studies are needed to determine criteria associated with grade of dysplasia/ malignancy, the clinical value of combining with current standard of care and cost-effectiveness (22).

Through the needle cystoscopy allows for direct visualization of the cyst content and inner walls by using a single operator cholangioscopy fiber optic probe. First, EUS guides a 19-gauge needle into the cyst, the needle stylet is removed and the probe advanced through the needle. This is a more invasive procedure and does carry the risk of pancreatitis, however, there have not been enough studies performed to determine the accuracy and risk profile of this procedure (22). Visualization of a tree-like branching of blood vessels may suggest a serous cystic neoplasm while an intracystic papilla-like structure suggests a mucinous cystic neoplasm (23). In a small study of 30 patients combining nCLE and cystoscopy, the sensitivity of cystoscopy alone was $90 \%$, nCLE $80 \%$ and combined $100 \%$ in 18 of the high-certainty patients (21). Furthermore, biopsies can be obtained from the wall of the cystic pancreatic lesions thus increasing the diagnostic yield of these types of lesions (24).

\section{Micro-forceps biopsy}

Micro-forceps biopsy is a technique where small caliber biopsy replaces the stylet in the $19 \mathrm{~g}$ EUS needle to sample the cyst wall under direct EUS visualization. Figure 2 demonstrates the EUS view of the moray microforceps sampling a cyst wall which was eventually found to be a mucinous cystic neoplasm. In a study comparing pancreatic cyst fluid analysis to micro-forceps biopsy 


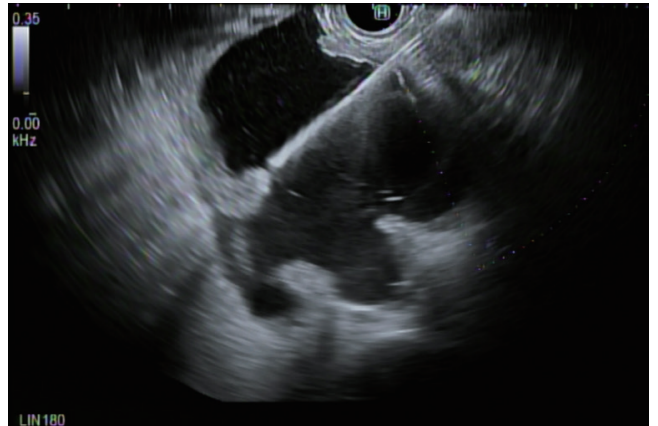

Figure 2 Moray needle microforceps biopsy of pancreatic cyst confirming mucinous cystic lesion. (Source: Ali M. Ahmed MD, 2019).

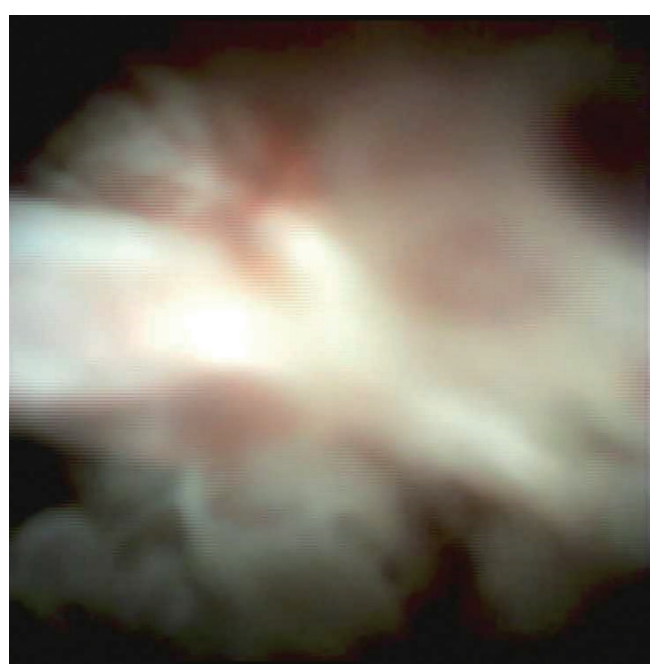

Figure 3 Pancreatoscopy depicting main duct IPMN. Spybite biopsy taken confirmed diagnosis of a mucinous lesion without dysplasia. (Source: Ali M. Ahmed MD, 2019). IPMN, intraductal papillary mucinous neoplasm.

of 48 cases the diagnostic yield was comparable $(72.9 \%$ vs. $75.0 \%$, respectively), however specific cyst diagnosis required cyst fluid analysis in a majority of cases (for GNAS or VHL mutation) (25). Another study evaluated 114 patients where the tissue acquisition $(83.3 \%$ vs. $37.7 \%)$ and diagnosis of mucinous lesions (53.5\% vs. 9.6\%) were greater in the samples achieved by micro-forceps biopsy (26). A study of 44 patients evaluated the additive effects of nCLE and micro-forceps biopsy (MFB) to EUS. The study found that the individual diagnostic yield for the EUS was $34.1 \%$, MFB $75.0 \%$ and nCLE $84.1 \%$ when compared to EUS as the reference standard $(\mathrm{P}<0.05)$.
However when all modalities were combined the diagnostic yield $93.2 \%$ although the $\mathrm{P}$ value was not significant. There may be a role for combining nCLE with MFB to further stratify pancreatic cysts in challenging cases (27).

\section{Single operator pancreatoscopy}

The advancement of digital cholangiopancreatoscopy has made per-oral pancreatoscopy a method to evaluate main duct IPMN with the ability to visualize papillary growths of IPMN in the MPD and perform targeted tissue acquisition to sample neoplastic cells (28). Figure 3 represents the peroral endoscopic pancreatoscopy images of a main duct IPMN.

\section{Contrast-harmonic enbanced endoscopic ultrasound (CH-EUS)}

Harmonic imaging uses microbubble ultrasound contrast agents (UCAs) that is injected intravenously and present a pure intravascular distribution allowing for real-time scanning with higher resolution than other imaging and visualization of pancreatic parenchyma perfusion. UCAs remain in the intravascular space and are eliminated by the lungs in exhaled air. A few studies have shown CH-EUS to be a promising noninvasive method to visualize and differentiate malignant from benign lesions (29-31). CH-EUS may define the inner structure of the pancreatic cysts by visualizing small septa or mural nodules and may assist in both the differential diagnosis and identifying high-risk features. Furthermore, when used in combination, it was shown to increase the sensitivity of EUS-FNA from $92 \%$ to $100 \%$ (31).

\section{Artificial intelligence (AI)}

Artificial Intelligence by deep learning has been studied in differentiating malignant from benign cystic lesions. By using a deep learning method, AI can possibly exclude the bias generated by human judgement. In a retrospective study 85 patients who underwent pancreatic cystic fluid analysis were also analyzed for malignant potential by AI. CEA, CA 19-9, CA 125, amylase in the fluid cyst and cytology were placed into the AI algorithm and malignant potential calculated. Sensitively and specificity for diagnosing malignant lesions was $95.7 \%$ and $91.9 \%$, respectively. However, this study was limited by sample size and cases were excluded if there was incorrect data or there was an item missing as AI cannot analyze without complete 
data (32).

\section{Current therapeutic options}

IPMNs and MCNs carry the highest malignant potential of all pancreatic cysts. Certain features within these cysts have an increased risk for developing into high-grade dysplasia or pancreatic adenocarcinoma. The definitive treatment is surgical resection however, cyst surgical excision carries a high morbidity and occasional mortality. A review by a multidisciplinary group who specialize in pancreatic cysts and pancreatic cancer is advised to review factors such as a patient's comorbidities, the type of surgery needed to remove the cyst and the estimated morbidity and mortality associated with the procedure. Concerning features are obstructive jaundice, an elevated CA 19-9, presence of a mural nodule, MPD dilation of $>6 \mathrm{~mm}$, large cyst diameter of $>3 \mathrm{~cm}$, rapid increase in cyst size, the presence of highgrade dysplasia or adenocarcinoma (4).

\section{Watchful waiting}

If an asymptomatic pancreatic cyst lacks concerning features, a watchful waiting approach can be used. There are different methods for surveillance used based on AGA and ACR guidelines as detailed above $(1,5)$. Once in a surveillance program, cyst size guides screening intervals for the presumed IPMNs and MCNs. Surveillance should stop if a patient is no longer a surgical candidate and it should be reassessed in those aged $>75$ years (4).

\section{Surgical excision}

The criteria for surgical indication in IPMNs and MCNs are based on IAP 2017 and European 2018 guidelines $(6,33)$. High-risk stigmata with indications for surgery in all patients eligible for the procedure include cyst-related jaundice, presence of vascularized mucinous neoplasm or solid component and a cytology suggesting malignancy. Surgery is also recommended if the MPD is $>1 \mathrm{~cm}$ and there also is a high suspicion of main duct or mixed IPMN. In IMPNs and MCNs, the entire tumor should be resected with lymphadenectomy and negative resection margins due to a high risk for malignancy. The remnant pancreas will require continued surveillance (34).

\section{Emerging therapeutic options}

\section{Endoscopic ultrasound guided fine needle injection (EUS-FNI)}

EUS-FNI is a minimally invasive approach used to deliver specific chemotherapeutic agents or other anti-tumor agents in those with pancreatic cysts and local control of tumor growth if the lesion is non-resectable. Agents used in cysts include ethanol and chemotherapeutic agents such as paxitaxel (35). In a study of 25 patients with cystic lesions, ethanol lavage was performed via EUS-FNI and $35 \%$ of patients had complete resolution of their cysts. Furthermore, five who underwent resection showed epithelial ablation. In another study 14 patients received both ethanol and paclitaxel injections for cystic tumors of the pancreas with a complete cystic tumor resolution in 11 patients and partial resolution in 2 at a median of 9 months. In both studies, there were minimal side effects with only one patient having acute pancreatitis $(36,37)$. However, follow-up guidelines with specific surveillance intervals post successful ethanol ablation are needed (38). A recent prospective study randomized 10 patients with known mucinous pancreatic cysts to lavage with ethanol or normal saline and treated the lesions with a combination of paclitaxel and gemcitabine. This study showed similar ablation rates between the ethanol ablation group and the ethanol-free arm suggesting ethanol may not be required for cyst ablation. However, larger, randomized control trials with longer follow-ups are needed to assess effectiveness and feasibility (39).

\section{Conclusions}

The diagnosis and treatment of pancreatic cysts remains challenging. The frequent detection of a pancreatic cyst is becoming more common due to new advanced imaging modalities and the frequency of their use in clinical practice. Management decision for asymptomatic pancreatic cysts must involve a multidisciplinary team and balance risk for malignancy with the complications of pancreatic surgery (4). On the other hand, PDAC is a devastating disease with a very poor prognosis due to late diagnosis (3). Opportunities to identify and reduce pre-cancer lesions must be aggressively pursued. We hope that a multimodal approach leveraging a variety of novel devices and 
technologies will streamline the approach and management of pancreatic cysts. Most studies to date are retrospective or lacking statistical power due to a small sample size. Further prospective randomized control trials are needed to determine the optimal management and treatment for patient with pancreatic cysts.

\section{Acknowledgments}

Funding: None.

\section{Footnote}

Provenance and Peer Review: This article was commissioned by the Guest Editor (Amy Tyberg) for the series "Innovation in Endoscopy" published in Translational Gastroenterology and Hepatology. The article has undergone external peer review.

Conflicts of Interest: Both authors have completed the ICMJE uniform disclosure form (available at https://tgh.amegroups. com/article/view/10.21037/tgh-2020-09/coif). The series "Innovation in Endoscopy" was commissioned by the editorial office without any funding or sponsorship. The authors have no other conflicts of interest to declare.

Ethical Statement: The authors are accountable for all aspects of the work in ensuring that questions related to the accuracy or integrity of any part of the work are appropriately investigated and resolved.

Open Access Statement: This is an Open Access article distributed in accordance with the Creative Commons Attribution-NonCommercial-NoDerivs 4.0 International License (CC BY-NC-ND 4.0), which permits the noncommercial replication and distribution of the article with the strict proviso that no changes or edits are made and the original work is properly cited (including links to both the formal publication through the relevant DOI and the license). See: https://creativecommons.org/licenses/by-nc-nd/4.0/.

\section{References}

1. Vege SS, Ziring B, Jain R, et al. American gastroenterological association institute guideline on the diagnosis and management of asymptomatic neoplastic pancreatic cysts. Gastroenterology 2015;148:819-22; quize $12-3$.
2. de Jong K, Nio CY, Hermans JJ, et al. High prevalence of pancreatic cysts detected by screening magnetic resonance imaging examinations. Clin Gastroenterol Hepatol 2010;8:806-11.

3. De Angelis C, Brizzi RF, Pellicano R. Endoscopic ultrasonography for pancreatic cancer: current and future perspectives. J Gastrointest Oncol 2013;4:220-30.

4. Elta GH, Enestvedt BK, Sauer BG, et al. ACG Clinical Guideline: Diagnosis and Management of Pancreatic Cysts. Am J Gastroenterol 2018;113:464-79.

5. Megibow AJ, Baker ME, Morgan DE, et al. Management of Incidental Pancreatic Cysts: A White Paper of the ACR Incidental Findings Committee. J Am Coll Radiol 2017;14:911-23.

6. Tanaka M, Fernández-Del Castillo C, Kamisawa T, et al. Revisions of international consensus Fukuoka guidelines for the management of IPMN of the pancreas. Pancreatology 2017;17:738-53.

7. Al-Hawary MM, Francis IR, Chari ST, et al. Pancreatic ductal adenocarcinoma radiology reporting template: consensus statement of the society of abdominal radiology and the american pancreatic association. Gastroenterology 2014;146:291-304.e1.

8. Pongpornsup S, Piyapittayanan S, Charoensak A. MDCT imaging findings for characterization pancreatic cystic lesion: differentiation between benign and malignant pattern. J Med Assoc Thai 2011;94:369-78.

9. Correa-Gallego C, Ferrone CR, Thayer SP, et al. Incidental pancreatic cysts: do we really know what we are watching? Pancreatology 2010;10:144-50.

10. Moris M, Bridges MD, Pooley RA, et al. Association Between Advances in High-Resolution Cross-Section Imaging Technologies and Increase in Prevalence of Pancreatic Cysts From 2005 to 2014. Clin Gastroenterol Hepatol 2016;14:585-593.e3.

11. Barresi L, Tarantino I, Granata A, et al. Pancreatic cystic lesions: How endoscopic ultrasound morphology and endoscopic ultrasound fine needle aspiration help unlock the diagnostic puzzle. World J Gastrointest Endosc 2012;4:247-59.

12. Rockacy M, Khalid A. Update on pancreatic cyst fluid analysis. Ann Gastroenterol 2013;26:122-7.

13. Thiruvengadam N, Park WG. Systematic Review of Pancreatic Cyst Fluid Biomarkers: The Path Forward. Clin Transl Gastroenterol 2015;6:e88.

14. van der Waaij LA, van Dullemen HM, Porte RJ. Cyst fluid analysis in the differential diagnosis of pancreatic cystic lesions: a pooled analysis. Gastrointest Endosc 
2005;62:383-9.

15. Khalid A, Zahid M, Finkelstein SD, et al. Pancreatic cyst fluid DNA analysis in evaluating pancreatic cysts: a report of the PANDA study. Gastrointest Endosc 2009;69:1095-102.

16. Anderson MA, Kwon RS, Scheiman JM. PANDA cystfluid analysis: eats, shoots and leaves? Gastrointest Endosc 2009;69:1103-5.

17. Springer S, Wang Y, Dal Molin M, et al. A combination of molecular markers and clinical features improve the classification of pancreatic cysts. Gastroenterology 2015;149:1501-10.

18. Kantsevoy SV, Adler DG, Conway JD, et al. Confocal laser endomicroscopy. Gastrointest Endosc 2009;70:197-200.

19. Fugazza A, Gaiani F, Carra MC, et al. Confocal Laser Endomicroscopy in Gastrointestinal and Pancreatobiliary Diseases: A Systematic Review and Meta-Analysis. Biomed Res Int 2016;2016:4638683.

20. Konda VJ, Meining A, Jamil LH, et al. A pilot study of in vivo identification of pancreatic cystic neoplasms with needle-based confocal laser endomicroscopy under endosonographic guidance. Endoscopy 2013;45:1006-13.

21. Nakai Y, Iwashita T, Park DH, et al. Diagnosis of pancreatic cysts: EUS-guided, through-the-needle confocal laserinduced endomicroscopy and cystoscopy trial: DETECT study. Gastrointest Endosc 2015;81:1204-14.

22. Alvarez-Sánchez MV, Napoléon B. New horizons in the endoscopic ultrasonography-based diagnosis of pancreatic cystic lesions. World J Gastroenterol 2018;24:2853-66.

23. Chai N, Feng J, Guo Y, et al. Preliminary study of singleoperator cholangioscopy for diagnosing pancreatic cystic lesions. Gastrointest Endosc 2017;86:208-18.

24. Aparicio JR, Martínez J, Niveiro M, et al. Direct intracystic biopsy and pancreatic cystoscopy through a 19-gauge needle EUS (with videos). Gastrointest Endosc 2010;72:1285-8.

25. Zhang ML, Arpin RN, Brugge WR, et al. Moray Micor Forceps Biopsy Improves the Diagnosis of Specific Pancreatic Cysts. Cancer Cytopathol 2018;126:414-20.

26. Yang D, Trindade A, Yachimski P, et al. Histologic Analysis of Endoscopic Ultrasound-Guided Through The Needle Microforceps Biopsies Accurately Identifies Mucinous Pancreatic Cysts. Clin Gastroenterol Hepatol 2019;17:1587-96.

27. Cheesman AR, Zhu H, Liao X, et al. Impact of EUSguided microforceps biopsy sampling and needle-based confocal laser endomicroscopy on the diagnostic yield and clinical management of pancreatic cystic lesions. Gastrointest Endosc 2020;91:1095-104.
28. Tanaka M. Current roles of endoscopy in the management of intraductal papillary mucinous neoplasm of the pancreas. Dig Endosc 2015;27:450-7.

29. Alvarez-Sánchez MV, Napoléon B. Contrast-enhanced harmonic endoscopic ultrasound imaging: basic principles, present situation and future perspectives. World J Gastroenterol 2014;20:15549-63.

30. Napoleon B, Alvarez-Sanchez MV, Gincoul R, et al. Contrast-enhanced harmonic endoscopic ultrasound in solid lesions of the pancreas: results of a pilot study. Endoscopy 2010;42:564-70.

31. Kitano M, Kudo M, Yamao K, et al. Characterization of small solid tumors in the pancreas: the value of contrastenhanced harmonic endoscopic ultrasonography. Am J Gastroenterol 2012;107:303-10.

32. Kurita Y, Kuwahara T, Hara K, et al. Diagnostic ability of artificial intelligence using deep learning analysis of cyst fluid in differentiating malignant from benign pancreatic cystic lesions. Sci Rep 2019;9:6893.

33. Pancreas ESGoCTot. European evidence-based guidelines on pancreatic cystic neoplasms. Gut 2018;67:789-804.

34. Perri G, Marchegiani G, Frigerio I, et al. Management of Pancreatic Cystic Lesions. Dig Surg 2020;37:1-9.

35. Verna EC, Dhar V. Endoscopic ultrasound-guided fine needle injection for cancer therapy: the evolving role of therapeutic endoscopic ultrasound. Therap Adv Gastroenterol 2008;1:103-9.

36. Gan SI, Thompson CC, Lauwers GY, et al. Ethanol lavage of pancreatic cystic lesions: initial pilot study. Gastrointest Endosc 2005;61:746-52.

37. Oh HC, Seo DW, Lee TY, et al. New treatment for cystic tumors of the pancreas: EUS-guided ethanol lavage with paclitaxel injection. Gastrointest Endosc 2008;67:636-42.

38. DeWitt J, DiMaio CJ, Brugge WR. Long-term followup of pancreatic cysts that resolve radiologically after EUS-guided ethanol ablation. Gastrointest Endosc 2010;72:862-6.

39. Moyer MT, Dye CE, Sharzehi S, et al. Is alcohol required for effective pancreatic cyst ablation? The prospective randomized CHARM trial pilot study. Endosc Int Open 2016;4:E603-7.

doi: 10.21037/tgh-2020-09

Cite this article as: Shipley LC, Ahmed AM. New and emerging technology in the diagnosis and treatment of pancreatic cysts. Transl Gastroenterol Hepatol 2022;7:15. 


\section{Pancreatic Cyst on Imaging}

Most consistent with

\begin{tabular}{|l|}
\hline Serous Cyst Adenoma \\
1. Refer to surgical \\
clinic to review \\
symptoms \\
2. Resect if \\
symptomatic \\
3. No further imaging
\end{tabular}

\begin{tabular}{|l|}
\hline Pseudocyst \\
1. \\
Correlate with \\
history \\
2.
\end{tabular}

Malignancy, Solid

Pseudopapillary

Neoplasm, or Mucinous

Cystic Neoplasm

1. Refer to surgery

\section{Asymptomatic BD-IPMN Algorithm}

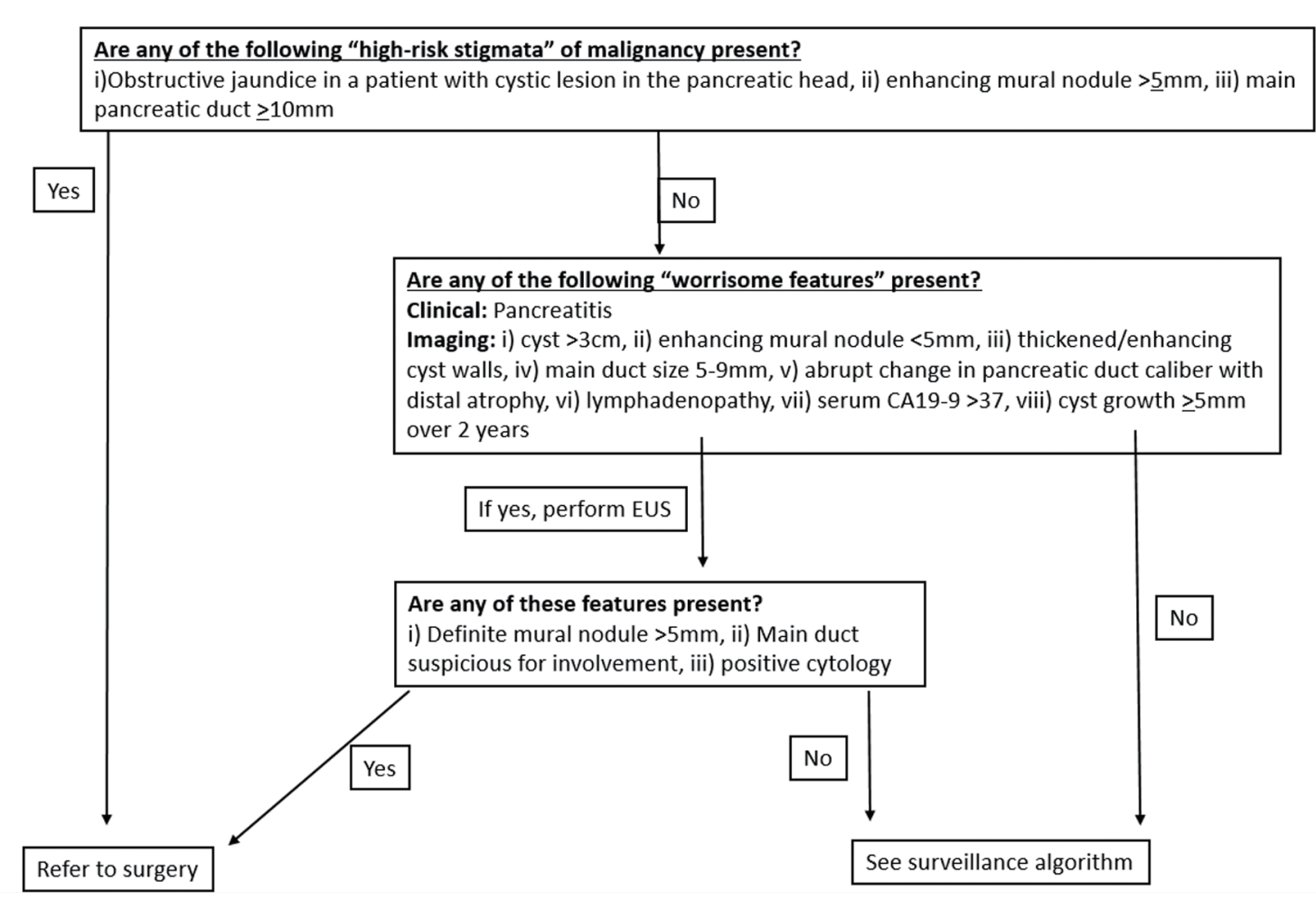

Surveillance

Size of largest cyst

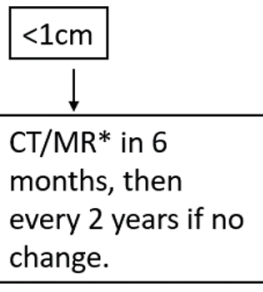

\begin{tabular}{|l|}
\hline CT/MR* in 6 \\
months $\times 2$, then \\
yearly $x 2$, then \\
every 2 years if \\
stable.
\end{tabular}

IPMN

1. For BD-IPMN:

a) If asymptomatic see BD-IPMN algorithm

b) If symptomatic then refer to surgery

2. For MD-IPMN or mixed

a) If duct $\geq 5 \mathrm{~mm}$ then MRCP and

Pancreatoscopy

b) If duct $<5 \mathrm{~mm}$ then EUS 